\title{
GENETIC ANALYSIS OF ACHENE YIELD IN SUNFLOWER (Helianthus annuus L.) THROUGH PYRAMIDING OF ASSOCIATED GENETIC FACTORS
}

\author{
Mubashir Manzoor ${ }^{1, *}$, Hafeez Ahmad Sadaqat', Muhammad Hammad Nadeem Tahir ${ }^{1}$ and \\ Bushra Sadia ${ }^{2}$
}

\author{
${ }^{1}$ Department of Plant Breeding and Genetics, University of Agriculture, Faisalabad, Pakistan; ${ }^{2}$ Centre of Agricultural \\ Biotechnology and Biochemistry, University of Agriculture, Faisalabad, Pakistan. \\ "Corresponding author's e-mail: mubashir.manzoor@uaf.edu.pk
}

\begin{abstract}
Over the years, the cultivation of sunflower has emerged as a popular cash crop and the area under it has increased during the last decade in Pakistan because of its profitability than other competing crops. Besides, this crop needs little water, while its harvesting time is short and it can easily be cultivated between two major crops. The present study was conducted to assess the genetic potential of sunflower accessions for achene yield, achene weight and achene oil contents. The breeding material consisted of more than 102 sunflower accessions out of which 9 were selected. Morphological, quality and yield related traits recorded were; achene yield per plant, 1000 achene weight, oil contents, head diameter, leaf area, plant height, number of leaves per plant, days to $50 \%$ flowering, days taken to maturity, days to complete flowering, intermodal length, stem girth, palmitic acid, stearic acid, oleic acid, linoleic acid and protein contents.
\end{abstract}

Keywords: Sunflower, pyramiding, heterosis, combining ability, achene yield, oil contents.

\section{INTRODUCTION}

Sunflower hybrids having higher genetic potential for achene yield and oil contents are required to fulfill the gap between supply and demand of vegetable oil all over the world. Like other cross pollinated crops sunflower hybrids are popular due to high yield and uniformity. The foremost prerequisite of hybrid development is the manifestation of heterosis (Skoric et al., 2000; Hladni et al., 2007). It is vital to find out the best parents with good combining abilities for hybridization and superior combinations among them (Gangappa et al., 1997). Higher general combining ability (GCA) effects show additive gene effects whereas higher specific combining ability (SCA) effects indicate dominance gene effects. Epistatic effect may also be found due to the non-significant of GCA and SCA effects (Fehr, 1993).

Important traits which directly contribute towards achene yield are plant height, 1000 achene weight, head diameter, days to $50 \%$ flowering and days taken to maturity (Kaya et al., 2006; Mijic et al., 2006; Machikowa and Saetang, 2008). Short statured plants having thick stems enhance crop stand and promote stability and increase number of plants per hectare (Suzer and Atakisi, 1993). Hybrids having shorter plant height have same yield potential as compared to regular height hybrids (Schneiter, 1992; Velasco et al., 2003a) and are impervious to lodging and stalk breaking (Fick et al., 1985). Number of leaves per plant, days taken to $50 \%$ flowering and days taken to maturity are also important factors for achene yield (Ghaffari et al., 2011; Jockovic et al., 2012). The objective of this study was to develop hybrids of sunflower having high achene yield with higher oil contents percentage by pyramiding the studied traits through classical breeding. The effect of general combining abilities of inbred lines and specific combining abilities of $F_{1}$ hybrids, heterosis, heterobeltiosis, commercial heterosis, correlations and path-coefficient analysis were performed.

\section{MATERIALS AND METHODS}

The research work was conducted in the research area of the Department of Plant Breeding and Genetics, University of Agriculture, Faisalabad, situated in the rolling flat plains of North East Punjab. It is between longitudes $73^{\circ}-06$ east, latitude $30^{\circ}-26$ north and altitude is $184.4 \mathrm{~m}$. It possesses loamy soil in field with the $0.53 \%$ organic content. Total 102 accessions were sown in field in triplicated randomized complete block design having row to row and plant to plant distances 75 and $25 \mathrm{~cm}$, respectively. Screening was done for high achene yield, weight and oil contents. The breeding materials were selected on the basis of the highest performance. The total selected populations consisted of 9 lines, three for each of high achene yield $(\mathrm{Y}), 1000$ achene weight $(\mathrm{W})$ and achene oil contents $(\mathrm{O})$. Data regarding other yield attributed traits such as; plant height, head diameter, days taken to $50 \%$ flowering, number of leaves per plant, days taken to maturity, leaf area, days taken to complete flowering, internodal length, stem girth, achene oil profile (oleic acid, linoleic acid and linolenic acid) and protein contents were also measured. The fatty acid profile and protein contents were measured by NIR (System 6500, 
Software: WINISS-Version 1.02A). The selected 9 lines were sown in the field as crossing block and crossing was done in line $\times$ tester fashion (Kempthorne, 1957) of $\left(\mathrm{Y}_{1-3} \times\right.$ $\left.\mathrm{W}_{1-3}\right),\left(\mathrm{Y}_{1-3} \times \mathrm{O}_{1-3}\right)$ and $\left(\mathrm{W}_{1-3} \times \mathrm{O}_{1-3}\right)$ generating $27 \mathrm{~F}_{1}$ hybrids. The highest performing $\mathrm{F}_{1}$ hybrids were taken into account for double crosses to pyramid $\mathrm{Y}, \mathrm{W}$ and $\mathrm{O}$ traits through controlled pollinations. The genetic materials were used for genetic variability following Steel et al. (1997), genetic analysis i.e., general combining ability (GCA) and specific combining ability and heterosis analysis.

\section{RESULTS AND DISCUSSION}

All the accessions showed significant differences (Table 1-2) for the achene yield, 1000 achene weight and oil contents, suggesting that selection should be preferred in this germplasm. Range of mean values of plant height (169 to $276 \mathrm{~cm}$ ), achene yield per plant (31 to $70 \mathrm{~g}$ ), oil contents ( 23.99 to $52 \%$ ), head diameter (16 to $23 \mathrm{~cm}$ ), 1000 achene weight (46 to $80 \mathrm{~g}$ ), days to $50 \%$ flowering (44 to 58), number of leaves per plant (16 to 28 ), days taken to maturity (98 to 100), leaf area (463 to $907 \mathrm{~cm}^{2}$ ), days to complete flowering (71 to 85), internodal length (4 to $7 \mathrm{~cm}$ ) and stem girth $(3$ to $5 \mathrm{~cm})$ indicated that significant differences were found among inbred lines, $F_{1}, F_{2}$ and double cross hybrids. The combining ability analysis (Table 4-7) showed variable direction and magnitude of general combining ability (GCA) effects among lines and testers and specific combining ability effects (SCA) among hybrids (Ghaffari et al., 2011). The line A8 showed highest GCA for achene yield per plant and 1000 achene weight, line A22 showed highest GCA for oil contents and line A14.1 showed highest GCA for 1000 achene weight. The highest SCA was shown by crosses $\mathrm{A} 8 \times \mathrm{A} 14.1, \mathrm{~A} 8 \times \mathrm{A} 7.7$ and $\mathrm{A} 14.1 \times \mathrm{A} 7.7$ for oil contents and the highest SCA for achene yield was shown by $\mathrm{A} 8 \times \mathrm{A} 7.7$ and $\mathrm{A} 22 \times \mathrm{A} 2.5$. It is obvious from these results that these parental lines can be used in the development of hybrid seed for higher yield which can grow rapidly and establish a good stand. Most of the hybrids which made the best specific combinations for various characters involved either one or both of the parents with good general combining ability. Some of the hybrids with significant and positive specific combining ability involved the parents which were not good combiners for the traits studied. It showed the importance of non-additive genetic effects controlling these characters. The hybrid combinations with high mean, with favorable SCA estimates and involving at least one of the parents with high GCA, would tend to increase the concentration of favorable alleles and this is an appreciable situation to any breeder. Most of the hybrids with high SCA effects for various characters involved either one or both of the parents with good general combining ability indicating the preponderance of additive genetic effects. High SCA effects resulting from crosses between higher general combiners can be improved through early selection. High SCA effects resulting from low GCA combiners suggested that such crosses may be utilized for further improvement through single plant selection in the later generations.

Genetic components of variability: The results were further confirmed by computation of degree of dominance, additive variance and dominance variances (Table 3). Average degree of dominance $\left(\sqrt{ } \sigma^{2} \mathrm{D} / \sigma^{2} \mathrm{~A}\right)$ was greater than one for majority of the traits, while dominance variances were also greater than the additive variances for majority of the traits (Setty et al., 1977; Joksimovic et al., 2000). It became quite clear that over dominance was primarily controlling majority of the traits. Hence heterosis breeding could be effective in exploitation of the genetic material (Hladni et al., 2007).

Heterosis manifestation: Heterosis was observed among the hybrids over mid parent, better parents and standard hybrids for various yield and yield related traits. The perusal of Table 8 indicated that cross A22 $\times$ A 2.5 showed the highest mid parent heterosis, cross A18.6×A2.5 showed the highest better parent heterosis and cross $\mathrm{A} 8 \times \mathrm{A} 7.7$ showed highest commercial heterosis for achene yield per plant. Highest mid parent and better parent heterosis was shown by the cross A18.6×A13.1 while cross A8 $\times$ A14.1 showed highest commercial heterosis for 1000 achene weight. Highest mid parent heterosis was shown by the cross A18.6×A14.1 while cross A18.6×A22 showed highest better parent heterosis for oil contents. Highest commercial heterosis was shown by A22 $\times$ A2.5 for oil contents. Achene yield ranged from -26.42 to 14.21 for mid parent, -54.22 to 9.91 for better parent and 33.08 to 26.46 for commercial heterosis. Positive and significant heterosis over mid, better and commercial was observed for 1000 achene weight for crosses A18.6×A2.5 and A18.6×A13.1. 1000 Achene weight ranged from -2.07 to 12.16 for mid parent, -25.36 to 9.32 for better parent and 30.44 to 7.89 for commercial heterosis. Positive and significant heterosis over mid, better and commercial was observed for cross $\mathrm{A} 8 \times \mathrm{C} 2.19$. Oil contents ranged from 12.09 to 19.00 for mid parent, -30.23 to 17.21 for better parent and 11.80 to 36.07 for commercial heterosis.

Table 1. Mean squares associated with achene yield, 1000 achene weight and oil contents.

\begin{tabular}{lcccc}
\hline S.O.V & DF & Achene Yield & 1000 Achene Weight & Oil Contents \\
\hline Replication & 2 & 15.78 & 28.55 & 33.48 \\
Genotype & 101 & $176.42^{* *}$ & $344.83^{* *}$ & $11.72^{* *}$ \\
Error & 202 & 18.58 & 5.22 & 3.21 \\
\hline
\end{tabular}

$*=$ Significant at $5 \%$ probability level; $* *=$ Significant at $1 \%$ probability level 


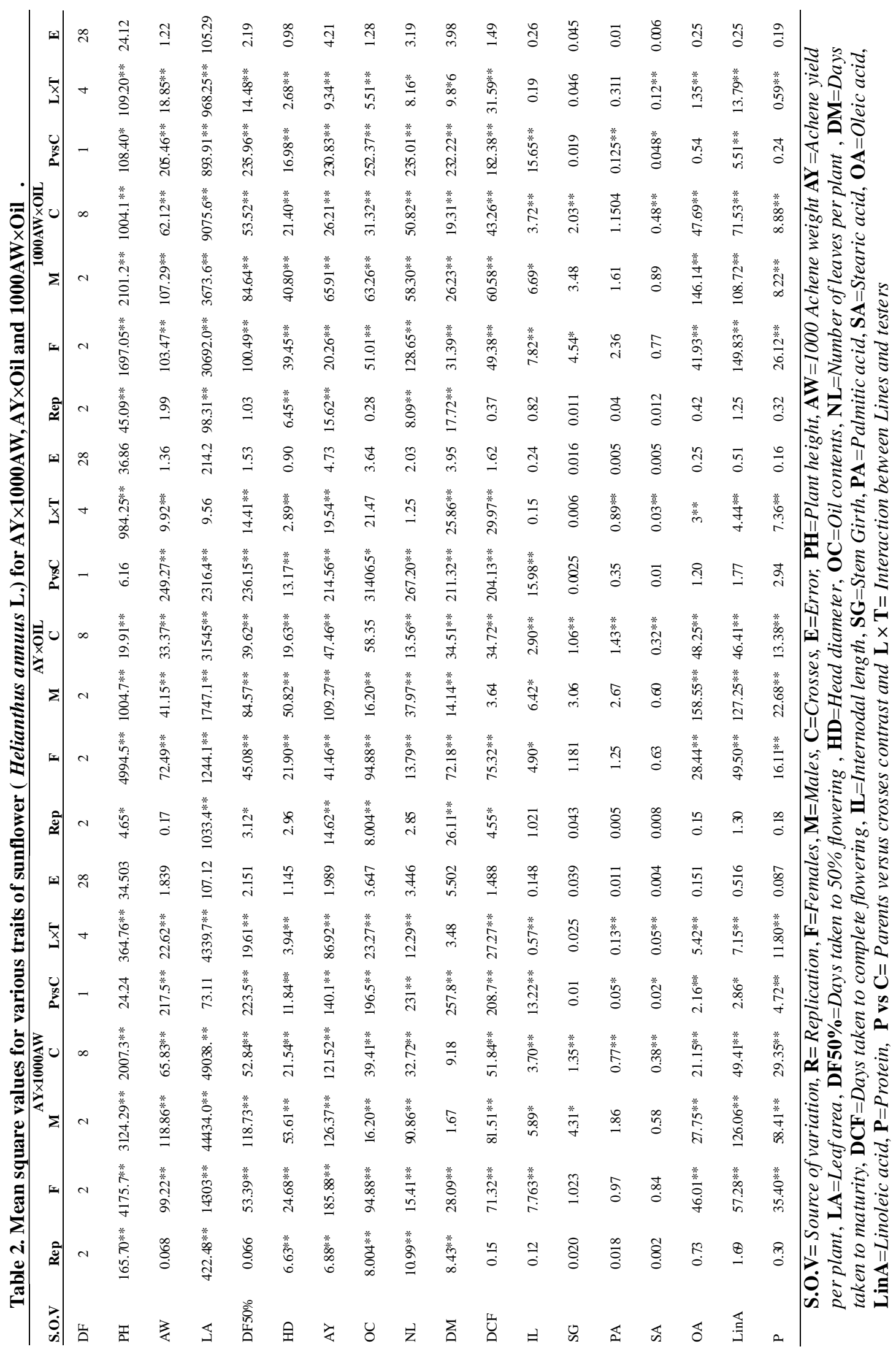


Table 3. Estimates of additive variance, dominance variance and degree of dominance in sunflower accessions

\begin{tabular}{|c|c|c|c|c|c|c|c|c|c|}
\hline \multirow{3}{*}{ Traits } & \multicolumn{9}{|c|}{ Genetic Components } \\
\hline & \multicolumn{3}{|c|}{$\sigma^{2} \mathbf{A}$} & \multicolumn{3}{|c|}{$\sigma^{2} D$} & \multicolumn{3}{|c|}{$\left(\sigma^{2} \mathrm{D} / \sigma^{2} \mathrm{~A}\right)^{1 / 2}$} \\
\hline & $\mathrm{AY} \times 1000 \mathrm{AW}$ & $\mathrm{AY} \times \mathrm{OIL}$ & $1000 \mathrm{AW} \times \mathrm{OIL}$ & $\mathrm{AY} \times 1000 \mathrm{AW}$ & $\mathrm{AY} \times \mathrm{OIL}$ & $1000 \mathrm{AW} \times \mathrm{OIL}$ & $\mathrm{AY} \times 1000 \mathrm{AW}$ & $\mathrm{AY} \times \mathrm{OIL}$ & $1000 \mathrm{AW} \times \mathrm{OIL}$ \\
\hline $\mathrm{PH}$ & 110.08 & 315.79 & 28.35 & 182.51 & 111.96 & 99.44 & 0.77 & 1.67 & 0.53 \\
\hline AW & 6.92 & 2.85 & 5.87 & 4.80 & 2.60 & 4.80 & 1.20 & 1.04 & 1.10 \\
\hline LA & 1410.85 & -68.21 & 287.65 & 4966.49 & 3503.96 & 900.81 & 0.53 & 0.13 & 0.56 \\
\hline DF50\% & 5.82 & 4.29 & 4.09 & 3.69 & 2.80 & 4.33 & 1.25 & 1.23 & 0.97 \\
\hline HD & 0.93 & 0.66 & 0.56 & 1.95 & 1.85 & 2.08 & 0.69 & 0.59 & 0.51 \\
\hline AY & 28.3 & 4.93 & 1.7 & 3.84 & 3.10 & 1.87 & 1.28 & 1.26 & 0.95 \\
\hline $\mathrm{OC}$ & 6.54 & -1520.15 & 1.4 & 1.79 & 4.09 & 2.86 & 1.91 & 1.35 & 0.69 \\
\hline NL & 2.94 & -0.26 & 1.65 & 2.26 & 1.36 & 4.73 & 1.14 & 0.43 & 0.59 \\
\hline DM & -0.67 & 7.29 & 1.94 & 0.63 & 0.96 & 1.05 & 1.03 & 2.75 & 1.35 \\
\hline DCF & 8.59 & 9.44 & 10.01 & 2.73 & 0.52 & 1.30 & 1.77 & 4.26 & 2.77 \\
\hline IL & 0.14 & -0.03 & -0.02 & 0.34 & 0.30 & 0.39 & 0.64 & 0.31 & 0.22 \\
\hline SG & 0 & 0 & 0 & 0.14 & 0.11 & 0.22 & 0.17 & 0.17 & 0.03 \\
\hline PA & 0.04 & 0.29 & 0.1 & 0.07 & 0.05 & 0.09 & 0.75 & 2.40 & 1.05 \\
\hline SA & 0.01 & 0 & 0.03 & 0.03 & 0.03 & 0.03 & 0.57 & 0.51 & 1.00 \\
\hline $\mathrm{OA}$ & 1.75 & 0.91 & 0.36 & 1.74 & 5.02 & 5.14 & 1.00 & 0.42 & 0.26 \\
\hline $\operatorname{Lin} A$ & 2.21 & 1.3 & 4.51 & 4.69 & 4.66 & 6.41 & 0.68 & 0.52 & 0.83 \\
\hline $\mathrm{P}$ & 3.9 & 2.39 & 0.13 & 1.95 & 0.66 & 0.92 & 1.41 & 1.90 & 0.37 \\
\hline
\end{tabular}

$\mathbf{\sigma}^{2} \mathbf{A}=$ Dominance Variance, $\mathbf{\sigma}^{2} \mathbf{D}=$ Additive Variance, $\mathbf{P H}=$ Plant Height, $\mathbf{A W}=1000$ Achene Weight, LA=Leaf Area, $\mathbf{D F 5 0 \%} \%$ Days taken to $50 \%$ flowering. $\mathbf{H D}=$ Head diameter, $\mathbf{A Y}=$ Achene yield per plant, $\mathbf{O C}=$ Oil contents, $\mathbf{N L}=$ Number of leaves per plant, $\mathbf{D M}=$ Days taken to maturity, $\mathbf{D C F}=$ Days taken to complete flowering, $\mathbf{I L}=$ Internodal length, $\mathbf{S G}=$ Stem Girth, $\mathbf{P A}=$ Palmitic acid, $\mathbf{S A}=$ Stearic acid, $\mathbf{O A}=$ Oleic acid, $\mathbf{L i n} \mathbf{A}=$ Linoleic acid and $\mathbf{P}=$ Protein

Table 4. Estimation of general combining ability effects of sunflower crosses AY $1000 \mathrm{X}$ AW for yield and related traits

\begin{tabular}{|c|c|c|c|c|c|c|c|c|c|}
\hline \multirow{3}{*}{ Traits } & \multicolumn{8}{|c|}{ GCA effects } & \\
\hline & \multicolumn{3}{|c|}{$\mathrm{AY} \times 1000 \mathrm{AW}$} & \multicolumn{3}{|c|}{ AY $\times$ Oil } & \multicolumn{3}{|c|}{ 1000AW $\times$ Oil } \\
\hline & A8 & A10.11 & A18.6 & A8 & A10.11 & A18.6 & A14.1 & C2.19 & A22 \\
\hline $\mathrm{PH}$ & $-13.62 * *$ & $-11.20 * *$ & $24.83^{* *}$ & $-11.37 * *$ & $-15.71 * *$ & $27.08 * *$ & $-12.42 * *$ & -2.31 & $14.74 * *$ \\
\hline AW & $3.18 * *$ & 0.26 & $-3.443 * *$ & $2.86 * *$ & -0.05 & -2.80 & $2.24 * *$ & $-3.9 * *$ & $1.65^{* *}$ \\
\hline LA & $-74.28 * *$ & $-71.26 * *$ & $145.5 * *$ & -88.06 & $-45.459 * *$ & $133.57 * *$ & $49.10 * *$ & $15.46^{* *}$ & $-64.57 * *$ \\
\hline DF50\% & $2.23 * *$ & 0.37 & $-2.59 * *$ & $2.10 * *$ & 0.24 & $-2.34 * *$ & $2.70 * *$ & 1.03 & $-3.73 * *$ \\
\hline HD & $-1.85 * *$ & $1.33 * *$ & 0.52 & $-1.74 * *$ & $1.27 * *$ & 0.46 & 0.06 & $-2.12 * *$ & $2.06 * *$ \\
\hline AY & $3.33^{* *}$ & $1.84 * *$ & $-5.17 * *$ & $2.47 * *$ & -1.30 & -1.17 & $1.59 *$ & -1.38 & -0.21 \\
\hline $\mathrm{OC}$ & 0.33 & $-3.40 * *$ & $3.06 * *$ & 0.33 & -3.40 & 3.06 & 0.76 & $-2.67 * *$ & $1.90 * *$ \\
\hline NL & 0.62 & 0.88 & $-1.50 *$ & 0.27 & $1.07 *$ & $-1.35^{*}$ & 0.23 & $-3.89 * *$ & $3.65 * *$ \\
\hline DM & $2.03 * *$ & -0.89 & -1.14 & $2.49 * *$ & 0.57 & $-3.0 * *$ & $-2.14 * *$ & 0.90 & 1.24 \\
\hline $\mathrm{DCF}$ & $2.63 * *$ & 0.32 & $-2.96 * *$ & $2.68 * *$ & 0.37 & $-3.0 * *$ & $2.53 * *$ & $-2.07 * *$ & -0.46 \\
\hline IL & $-1.07 * *$ & $0.48 * *$ & $0.58 * *$ & $-0.85^{* *}$ & $0.37 *$ & $0.47 *$ & $-1.07 * *$ & $0.50 * *$ & $0.57 * *$ \\
\hline SG & $0.34 * *$ & -0.02 & $-0.32 * *$ & $0.33 * *$ & 0.05 & $-0.38 * *$ & $0.44 * *$ & $-0.81 * *$ & $0.37 * *$ \\
\hline PA & $0.28 * *$ & $-0.36 * *$ & $0.08 * *$ & $0.39 * *$ & -0.04 & $-0.35 * *$ & 0.04 & $-0.53 * *$ & $0.49 * *$ \\
\hline SA & $0.31^{* *}$ & -0.01 & $-0.29 * *$ & $0.30 * *$ & $-0.12 * *$ & $-0.17 * *$ & $0.27 * *$ & $-0.31 * *$ & 0.03 \\
\hline $\mathrm{OA}$ & $2.16^{* *}$ & $-2.34 * *$ & 0.18 & $2.05 * *$ & $-1.11 * *$ & $-0.93 * *$ & $1.69 * *$ & $-2.43 * *$ & $0.74 * *$ \\
\hline $\operatorname{Lin} A$ & $0.53 *$ & $-2.74 * *$ & $2.21 * *$ & 0.42 & $-2.52 * *$ & $2.10^{* *}$ & $1.42 * *$ & $-4.60 * *$ & $3.17 * *$ \\
\hline $\mathrm{P}$ & $0.58 * *$ & $-2.21 * *$ & $1.62 * *$ & $-1.11 * *$ & $-0.36^{*}$ & $1.48 * *$ & 0.25 & $-1.81 * *$ & 1.55 \\
\hline
\end{tabular}

$\mathbf{P H}=$ Plant height, $\mathbf{A W}=1000$ Achene weight $\mathbf{A Y}=$ Achene yield per plant, $\mathbf{L A}=$ Leaf area, $\mathbf{D F 5 0 \% = D a y s ~ t a k e n ~ t o ~} 50 \%$ flowering, $\mathbf{H D}=$ Head diameter, $\mathbf{O C}=$ Oil contents, $\mathbf{N L}=$ Number of leaves per plant, $\mathbf{D M}=$ Days taken to maturity, $\mathbf{D C F}=$ Days taken to complete flowering, $\mathbf{I L}=$ Internodal length, $\mathbf{S G}=$ Stem Girth, $\mathbf{P A}=$ Palmitic acid, $\mathbf{S A}=$ Stearic acid, $\mathbf{O A}=$ Oleic acid, LinA=Linoleic acid, $\mathbf{P}=$ Protein 
Table 5. Estimation of specific combining ability effects of sunflower crosses AY $1000 \mathrm{X}$ AW for yield and related traits.

\begin{tabular}{lccccccccc}
\hline Traits & A8XA14.1 & A8XC2.19 & A8XA22 & A10.11XA14.1 & A10.11XC2.19 & A10.11XA22 & A18.6XA14.1 & A18.6XC2.19 & A18.6XA22 \\
\hline PH & $6.67^{*}$ & -3.05 & -3.61 & $-14.67^{* *}$ & $7.44^{*}$ & $7.22^{*}$ & $8.00^{*}$ & -4.39 & -3.61 \\
AW & $-1.51^{*}$ & $2.13^{* *}$ & -0.623 & -0.51 & 1.46 & -0.95 & $2.02^{* *}$ & $-3.60^{* *}$ & $1.58^{*}$ \\
LA & $-13.39^{*}$ & -11.95 & $25.35^{* *}$ & $25.46^{* *}$ & $25.24^{* *}$ & $-50.70^{* *}$ & $-12.064^{*}$ & $-13.28^{*}$ & $25.35^{* *}$ \\
DF50\% & -1.44 & $2.00^{*}$ & -0.55 & -0.44 & 1.34 & -0.89 & 1.89 & $-3.34^{* *}$ & 1.45 \\
HD & 0.68 & $-1.47^{*}$ & 0.79 & -0.50 & 0.57 & -0.06 & -0.17 & 0.90 & -0.73 \\
AY & $-2.39^{* *}$ & $3.90^{* *}$ & -1.50 & -1.39 & $3.24^{* *}$ & -1.84 & $3.79^{* *}$ & $-7.14^{* *}$ & $3.31^{* *}$ \\
OC & 1.13 & -0.28 & -0.84 & $-3.60^{* *}$ & 1.91 & 1.69 & $2.46^{*}$ & -1.62 & -0.84 \\
NL & -1.37 & 1.31 & 0.05 & 0.17 & 1.19 & -1.36 & 1.20 & $-2.50^{*}$ & 1.30 \\
DM & -0.10 & 0.33 & -0.22 & -1.12 & 0.67 & 0.44 & 1.22 & -1.00 & -0.22 \\
DCF & $-1.60^{*}$ & $2.31^{* *}$ & 0.71 & -0.60 & $1.65^{*}$ & -1.04 & $2.20^{* *}$ & $-3.96^{* *}$ & $1.76^{*}$ \\
IL & 0.19 & $-0.53^{*}$ & 0.34 & $-0.08^{*}$ & 0.41 & -0.32 & -0.10 & 0.12 & -0.01 \\
SG & $-0.33^{* *}$ & 0.06 & -0.04 & 0.06 & -0.12 & 0.05 & -0.08 & $0.25^{*}$ & -0.03 \\
PA & -0.07 & $0.14^{*}$ & -0.80 & $0.13^{*}$ & $-0.28^{* *}$ & $0.24^{*}$ & $-0.17^{* *}$ & $0.14 * *$ & -0.06 \\
SA & -0.04 & 0.08 & -0.05 & -0.03 & 0.09 & -0.04 & 0.05 & $-0.17^{* *}$ & $0.09^{*}$ \\
OA & -0.45 & -0.38 & 0.46 & $0.74^{* *}$ & $0.86^{* *}$ & $-1.79^{* *}$ & -0.44 & $-0.48^{*}$ & $0.89^{* *}$ \\
LinA & -0.54 & $1.02^{*}$ & -0.51 & $1.09^{*}$ & $-2.05^{* *}$ & $1.02^{*}$ & -0.51 & $1.019^{*}$ & -0.51 \\
P & $-0.61^{* *}$ & $1.52^{* *}$ & $-0.66^{* *}$ & $1.93^{* *}$ & $-2.64^{* *}$ & $1.33^{* *}$ & $-0.78^{* *}$ & $1.32^{* *}$ & $-0.56^{* *}$ \\
\hline
\end{tabular}

$\mathbf{P H}=$ Plant height, $\mathbf{A W}=1000$ Achene weight $\mathbf{A Y}=$ Achene yield per plant, $\mathbf{L A}=$ Leaf area, $\mathbf{D F 5 0 \% = D a y s ~ t a k e n ~ t o ~ 5 0 \% ~}$ flowering, $\mathbf{H D}=$ Head diameter, $\mathbf{O C}=$ Oil contents, $\mathbf{N L}=$ Number of leaves per plant, $\mathbf{D M}=$ Days taken to maturity, $\mathbf{D C F}=$ Days taken to complete flowering, $\mathbf{I L}=$ Internodal length, $\mathbf{S G}=$ Stem Girth, $\mathbf{P A}=$ Palmitic acid, $\mathbf{S A}=$ Stearic acid, $\mathbf{O A}=$ Oleic acid, LinA=Linoleic acid, $\mathbf{P}=$ Protein

Table 6. Estimation of specific combining ability effects of sunflower crosses AY×OIL for yield and related traits.

\begin{tabular}{lclllccccc}
\hline Traits & A8XA2.5 & A8XA7.7 & A8XA13.1 & A10.11XA2.5 & A10.11XA7.7 & A10.11XA13.1 & A18.6XA2.5 & A18.6XA7.7 & A18.6XA13.1 \\
\hline PH & -6.75 & -5.31 & $12.06^{* *}$ & $12.17^{* *}$ & $11.95^{* *}$ & $-24.13^{* *}$ & -5.42 & -6.64 & $12.06^{* *}$ \\
AW & -1.19 & $1.50^{*}$ & -0.30 & -0.19 & 0.83 & -0.64 & 1.39 & $-2.33^{* *}$ & $0.94^{* *}$ \\
LA & 0.37 & 1.82 & -2.19 & -0.35 & -0.57 & 0.93 & -0.02 & -1.24 & 1.26 \\
DF50\% & -1.32 & $1.75^{*}$ & -0.434 & -0.32 & 1.09 & -0.76 & $1.64^{*}$ & $-2.85^{* *}$ & 1.20 \\
HD & 0.56 & $-1.24^{*}$ & 0.67 & -0.45 & 0.45 & -0.006 & -0.11 & 0.79 & -0.67 \\
AY & -1.54 & -0.09 & 1.64 & 1.75 & 1.53 & $-3.28^{* *}$ & -0.20 & -1.43 & 1.64 \\
OC & 1.13 & -0.28 & -0.84 & -3.60 & 1.91 & 1.69 & 2.46 & -1.62 & -0.84 \\
NL & -0.28 & 0.41 & -0.13 & 0.71 & -0.24 & -0.47 & -0.43 & -0.17 & 0.60 \\
DM & -1.57 & 2.26 & -0.68 & -0.57 & 1.59 & -1.02 & 2.15 & $-3.86^{* *}$ & 1.70 \\
DCF & $-1.65^{*}$ & $2.41^{* *}$ & -0.76 & -0.65 & $1.74^{*}$ & -1.09 & $2.30^{* *}$ & $-4.16^{* *}$ & $1.86^{*}$ \\
IL & -0.22 & 0.10 & 0.12 & 0.12 & 0.09 & -0.21 & 0.09 & -0.19 & 0.09 \\
SG & 0.03 & $-0.21^{*}$ & -0.01 & $0.18^{*}$ & -0.01 & -0.05 & -0.06 & 0.09 & 0.06 \\
PA & $0.36^{*}$ & $-0.18^{* *}$ & $-0.17^{* *}$ & $0.36^{* *}$ & $-0.18^{* *}$ & $-0.16^{* *}$ & $-0.72^{* *}$ & $0.36^{* *}$ & $0.36^{* *}$ \\
SA & -0.03 & -0.02 & 0.06 & 0.04 & 0.05 & $-0.13^{* *}$ & -0.03 & -0.04 & 0.07 \\
OA & $0.67^{*}$ & -0.43 & -0.53 & $0.66^{*}$ & -0.42 & -0.33 & $-1.33^{* *}$ & $0.67^{*}$ & 0.56 \\
LA & -0.41 & 0.81 & -0.40 & $0.84^{*}$ & $-1.62^{* *}$ & $0.85^{*}$ & -0.42 & $0.91^{*}$ & -0.40 \\
P & $1.04^{* *}$ & $1.12^{* *}$ & $-2.08^{* *}$ & $-0.52^{*}$ & $-0.53^{*}$ & $1.54^{* *}$ & $-0.522^{* *}$ & $-0.56^{*}$ & $1.13^{* *}$ \\
\hline
\end{tabular}

$\mathbf{P H}=$ Plant height, $\mathbf{A W}=1000$ Achene weight $\mathbf{A Y}=$ Achene yield per plant, $\mathbf{L A}=$ Leaf area, $\mathbf{D F 5 0 \% = D a y s ~ t a k e n ~ t o ~ 5 0 \%}$ flowering, $\mathbf{H D}=$ Head diameter, $\mathbf{O C}=$ Oil contents, $\mathbf{N L}=$ Number of leaves per plant, $\mathbf{D M}=$ Days taken to maturity, $\mathbf{D C F}=$ Days taken to complete flowering, $\mathbf{I L}=$ Internodal length, $\mathbf{S G}=$ Stem Girth, $\mathbf{P A}=$ Palmitic acid, $\mathbf{S A}=$ Stearic acid, $\mathbf{O A}=$ Oleic acid, LinA=Linoleic acid, $\mathbf{P}=$ Protein 
Table 7. Estimation of specific combining ability effects of sunflower crosses $1000 \mathrm{AW} \times$ Oil for yield and related traits.

\begin{tabular}{|c|c|c|c|c|c|c|c|c|c|}
\hline Traits & A14.1XA2.5 & A14.1XA7.7 & A14.1XA13.1 & C2.19XA2.5 & C2.19XA7.7 & C2.19XA13.1 & A22XA2.5 & A22XA7.7 & A22XA13.1 \\
\hline $\mathrm{PH}$ & 3.33 & -1.38 & -1.94 & $-7.99 *$ & 4.10 & 3.88 & 4.66 & -2.7 & -1.94 \\
\hline AW & 0.94 & -0.19 & -0.74 & $-3.21 * *$ & $1.72 *$ & $1.49 * *$ & $2.27 * *$ & $-1.52 * *$ & -0.74 \\
\hline LA & 11.47 & -5.45 & -6.01 & $12.47 *$ & -6.12 & -6.34 & $-23.94 * *$ & 11.58 & $12.35 * *$ \\
\hline DF50\% & -1.32 & 1.76 & -0.43 & -0.32 & 1.09 & -0.77 & 1.65 & $-2.85 * *$ & 1.2 \\
\hline HD & -0.41 & 0.71 & -0.3 & 0.38 & $-1.20 *$ & 0.82 & 0.03 & 0.49 & -0.52 \\
\hline AY & 0.42 & 0.06 & -0.48 & -2.17 & 1.19 & 0.97 & 1.75 & -1.26 & -0.48 \\
\hline $\mathrm{OC}$ & -1.16 & $1.44 *$ & -0.27 & 0.99 & $-1.55^{*}$ & 0.55 & 0.16 & 0.1 & -0.27 \\
\hline NL & 0.33 & 0.10 & -0.44 & -2.00 & 1.11 & 0.89 & 1.67 & -1.22 & -0.44 \\
\hline DM & 0.39 & 1.83 & -2.22 & -0.36 & -0.58 & 0.94 & -0.02 & -1.25 & 1.28 \\
\hline $\mathrm{DCF}$ & 1.44 & -0.44 & -1.00 & $-4.22 * *$ & $2.22 * *$ & $2.00 * *$ & $2.78 * *$ & $-1.7 *$ & -1 \\
\hline IL & -0.27 & 0.12 & 0.14 & 0.14 & 0.08 & -0.22 & 0.12 & -0.2 & 0.08 \\
\hline SG & 0.10 & -0.05 & -0.041 & -0.16 & 0.08 & 0.07 & 0.09 & -0.04 & -0.05 \\
\hline PA & $0.21 * *$ & $-0.10 * *$ & $-0.11 * *$ & $-0.42 * *$ & $0.21 * *$ & $0.23 * *$ & $0.22 * *$ & $-0.16^{*}$ & -0.14 \\
\hline SA & -0.03 & -0.04 & $0.13^{*}$ & $0.14 * *$ & $0.15^{* *}$ & $-0.26 * *$ & $-0.25 * *$ & $-0.027 * *$ & $0.13^{*}$ \\
\hline $\mathrm{OA}$ & $0.68 *$ & -0.22 & $-0.87 * *$ & $-0.89 * *$ & 0.44 & 0.43 & $0.89 * *$ & -0.22 & $-0.92 * *$ \\
\hline LA & $-0.71 *$ & $-0.75^{*}$ & $1.42 * *$ & $1.56 * *$ & $1.48 * *$ & $-2.85 * *$ & $-0.71 * *$ & $-0.75^{*}$ & $1.51 * *$ \\
\hline $\mathrm{P}$ & -0.14 & -0.18 & 0.29 & 0.26 & 0.32 & $-0.59 *$ & -0.14 & -0.12 & 0.23 \\
\hline
\end{tabular}

$\mathbf{P H}=$ Plant height, $\mathbf{A W}=1000$ Achene weight $\mathbf{A Y}=$ Achene yield per plant, $\mathbf{L A}=$ Leaf area, $\mathbf{D F 5 0 \% = D a y s ~ t a k e n ~ t o ~ 5 0 \%}$ flowering, $\mathbf{H D}=$ Head diameter, $\mathbf{O C}=$ Oil contents, $\mathbf{N L}=$ Number of leaves per plant, $\mathbf{D M}=$ Days taken to maturity, $\mathbf{D C F}=$ Days taken to complete flowering, $\mathbf{I L}=$ Internodal length, $\mathbf{S G}=$ Stem Girth, $\mathbf{P A}=$ Palmitic acid, $\mathbf{S A}=$ Stearic acid, $\mathbf{O A}=$ Oleic acid, $\operatorname{Lin} \mathbf{A}=$ Linoleic acid, $\mathbf{P}=$ Protein

Table 8. Heterosis $(\%)$ over mid-parent $\left(\mathrm{H}_{t}\right)$, better-parent $\left(\mathrm{H}_{\mathrm{b}}\right)$ and standard checks for achene yield, 1000 achene weight and oil contents.

\begin{tabular}{|c|c|c|c|c|c|c|c|c|c|c|c|}
\hline Crosses & $\mathbf{H}_{\mathrm{t}}$ & $\mathbf{H}_{\mathrm{b}}$ & FH-331 & Crosses & $\mathbf{H}_{\mathrm{t}}$ & $\mathbf{H}_{\mathrm{b}}$ & FH-331 & Crosses & $\mathbf{H}_{\mathrm{t}}$ & $\mathbf{H}_{\mathrm{b}}$ & FH-331 \\
\hline $\mathrm{A} 8 \times \mathrm{A} 14.1$ & $6.58 *$ & $-17.07 * *$ & $16.44 * *$ & $\mathrm{~A} 8 \times \mathrm{A} 14.1$ & 4.73 & -4.11 & $7.89 * *$ & $\mathrm{~A} 8 \times \mathrm{A} 14.1$ & $11.19 * *$ & 6.89 & $17.10 * *$ \\
\hline $\mathrm{A} 8 \times \mathrm{C} 2.19$ & $8.55^{*}$ & $-19.62 * *$ & $14.62 * *$ & $\mathrm{~A} 8 \times \mathrm{C} 2.19$ & $6.31 *$ & $3.20 *$ & $3.45 * *$ & $\mathrm{~A} 8 \times \mathrm{C} 2.19$ & $15.27 * *$ & $9.17 *$ & $10.91 * *$ \\
\hline $\mathrm{A} 8 \times \mathrm{A} 22$ & $8.24 *$ & $-24.06^{* *}$ & $11.46^{* * *}$ & $\mathrm{~A} 8 \times \mathrm{A} 22$ & 5.72 & 0.06 & $5.55^{* *}$ & $\mathrm{~A} 8 \times \mathrm{A} 22$ & $13.30 * *$ & $11.62 * *$ & $16.60 * *$ \\
\hline A $10.11 \times A 14.1$ & $10.25^{*}$ & $-9.12 *$ & $15.70 * *$ & A10.11×A14.1 & $7.49 *$ & $-6.75^{*}$ & $5.55^{* *}$ & $\mathrm{~A} 10.11 \times \mathrm{A} 14.1$ & $-12.09 * *$ & $-25.58 * *$ & $-11.80 * *$ \\
\hline $\mathrm{A} 10.11 \times \mathrm{C} 2.19$ & $9.54 *$ & $-15.05^{* *}$ & $11.12 * *$ & A10.11×C2.19 & $7.10^{*}$ & -1.73 & $-1.46^{*}$ & $\mathrm{~A} 10.11 \times \mathrm{C} 2.19$ & $17.25 * *$ & $17.21 * *$ & 6.21 \\
\hline $\mathrm{A} 10.11 \times \mathrm{A} 22$ & $9.83^{*}$ & $-18.74 * *$ & $8.27 *$ & $\mathrm{~A} 10.11 \times \mathrm{A} 22$ & $6.90 *$ & -4.42 & $1.31 *$ & $\mathrm{~A} 10.11 \times \mathrm{A} 22$ & $15.91 * *$ & 8.22 & $13.38 * *$ \\
\hline A18.6×A14.1 & $13.09 * *$ & -1.02 & $12.82 * *$ & A18.6×A14.1 & $9.40 * *$ & $-8.43^{* *}$ & $4.06^{* *}$ & A18.6×A14.1 & $19.00 * *$ & $17.15 * *$ & $26.24 * *$ \\
\hline A18.6×C2.19 & $-26.42 * *$ & $-54.22 * *$ & $-33.08 * *$ & A18.6×C2.19 & -2.07 & $-16.22^{* * *}$ & $-15.92 * *$ & A18.6×C2.19 & $16.71 * *$ & 8.61 & $14.82 * *$ \\
\hline $\mathrm{A} 18.6 \times \mathrm{A} 22$ & $12.93 * *$ & $-10.25^{* *}$ & 4.86 & A $18.6 \times A 22$ & $8.88 * *$ & $-6.13^{*}$ & -0.3 & $\mathrm{~A} 18.6 \times \mathrm{A} 22$ & $17.92 * *$ & $17.41 * *$ & $23.02 * *$ \\
\hline $\mathrm{A} 8 \times \mathrm{A} 2.5$ & 5.71 & -1.73 & $27.40 * *$ & $\mathrm{~A} 8 \times \mathrm{A} 2.5$ & $6.05^{*}$ & $-10.64 * *$ & $-17.92 * *$ & $\mathrm{~A} 8 \times \mathrm{A} 2.5$ & $9.54^{*}$ & -8.81 & $29.29 * *$ \\
\hline $\mathrm{A} 8 \times \mathrm{A} 7.7$ & $6.81 *$ & 4.77 & $32.03 * *$ & $\mathrm{~A} 8 \times \mathrm{A} 7.7$ & $7.26^{*}$ & -4.26 & $-11.12 * *$ & $\mathrm{~A} 8 \times \mathrm{A} 7.7$ & $13.04 * *$ & 3.6 & $23.97 * *$ \\
\hline $\mathrm{A} 8 \times \mathrm{A} 13.1$ & $6.85^{*}$ & -3.04 & $26.46 * *$ & $\mathrm{~A} 8 \times \mathrm{A} 13.1$ & $6.61 *$ & -2.21 & $-8.93 * *$ & $\mathrm{~A} 8 \times \mathrm{A} 13.1$ & $11.73^{* *}$ & -1.43 & $26.34 * *$ \\
\hline $\mathrm{A} 10.11 \times \mathrm{A} 2.5$ & $8.88^{*}$ & 5.29 & $26.84 * *$ & $\mathrm{~A} 10.11 \times \mathrm{A} 2.5$ & $9.65 * *$ & -0.90 & $-21.79 * *$ & $\mathrm{~A} 10.11 \times \mathrm{A} 2.5$ & $14.70 * *$ & $-8.45^{*}$ & $29.53 * *$ \\
\hline A10.11×A7.7 & $7.52 *$ & 5.89 & $29.82 * *$ & A10.11×A7.7 & $8.23 *$ & 2.48 & $-17.69 * *$ & A10.11×A7.7 & $-10.41 *$ & $-30.23 * *$ & -2.72 \\
\hline $\mathrm{A} 10.11 \times \mathrm{A} 13.1$ & -3.45 & $-10.37 * *$ & $14.73 * *$ & A10.11×A13.1 & $8.02 *$ & 5.02 & $-14.63 * *$ & $\mathrm{~A} 10.11 \times \mathrm{A} 13.1$ & $14.00 * *$ & -4.84 & $23.86 * *$ \\
\hline A18.6×A2.5 & $11.31 *$ & $9.91 *$ & $24.68 * *$ & $\mathrm{~A} 18.6 \times \mathrm{A} 2.5$ & $12.16^{* * *}$ & 5.74 & $-24.26 * *$ & A18.6×A2.5 & $16.48 * *$ & 1.57 & $36.04 * *$ \\
\hline A18.6×A7.7 & $8.39 *$ & 1.71 & $26.71 * *$ & A18.6×A7.7 & 2.86 & 1.05 & $-30.44 * *$ & A18.6×A7.7 & $14.35^{* *}$ & $7.21 *$ & $26.81 * *$ \\
\hline A18.6×A13.1 & $10.61 *$ & $9.56^{*}$ & $21.96 * *$ & A18.6×A13.1 & $10.35 * *$ & $9.32 * *$ & $-16.83 * *$ & A18.6×A13.1 & $15.98 * *$ & 5.55 & $31.41 * *$ \\
\hline $\mathrm{A} 14.1 \times \mathrm{A} 2.5$ & $7.55^{*}$ & $-8.89^{*}$ & $8.97 *$ & $\mathrm{~A} 14.1 \times \mathrm{A} 2.5$ & 5.43 & $-19.73 * *$ & $-5.93 * *$ & $\mathrm{~A} 14.1 \times \mathrm{A} 2.5$ & $9.66^{*}$ & -4.44 & $32.13 * *$ \\
\hline A14.1×A7.7 & $8.75^{*}$ & $-12.47 * *$ & $16.13 * *$ & $\mathrm{~A} 14.1 \times \mathrm{A} 7.7$ & $6.56^{*}$ & $-13.49 * *$ & -0.4 & $\mathrm{~A} 14.1 \times \mathrm{A} 7.7$ & $13.02 * *$ & $7.76^{*}$ & $27.24 * *$ \\
\hline A14.1×A13.1 & $8.99 *$ & -4.74 & $7.46^{*}$ & A14.1×A13.1 & 5.99 & $-11.47 * *$ & $1.38 *$ & A14.1×A13.1 & $11.78 * *$ & 2.82 & $29.43 * *$ \\
\hline $\mathrm{C} 2.19 \times \mathrm{A} 2.5$ & 1.05 & $-22.19 * *$ & -2.14 & $\mathrm{C} 2.19 \times \mathrm{A} 2.5$ & -3.63 & $-25.36^{* *}$ & $-25.03 * *$ & $\mathrm{C} 2.19 \times \mathrm{A} 2.5$ & $15.13 * *$ & $-7.86 *$ & $29.91 * *$ \\
\hline $\mathrm{C} 2.19 \times \mathrm{A} 7.7$ & $9.69 *$ & $-16.33^{* *}$ & $13.25 * *$ & $\mathrm{C} 2.19 \times \mathrm{A} 7.7$ & $7.47 *$ & $-7.03 *$ & $-6.75 * *$ & $\mathrm{C} 2.19 \times \mathrm{A} 7.7$ & 5.51 & $-11.41 * *$ & $12.12 * *$ \\
\hline $\mathrm{C} 2.19 \times \mathrm{A} 13.1$ & $10.65^{*}$ & $-7.98 *$ & 4.60 & $\mathrm{C} 2.19 \times \mathrm{A} 13.1$ & $7.29 *$ & -4.50 & $-4.23 * *$ & $\mathrm{C} 2.19 \times \mathrm{A} 13.1$ & $14.48 * *$ & -4.23 & $24.31 * *$ \\
\hline $\mathrm{A} 22 \times \mathrm{A} 2.5$ & $14.21 * *$ & $-9.90^{*}$ & $8.12 *$ & $\mathrm{~A} 22 \times \mathrm{A} 2.5$ & $10.25^{* *} *$ & $-10.85^{* *}$ & $-4.76^{* * *}$ & $\mathrm{~A} 22 \times \mathrm{A} 2.5$ & $16.94 * *$ & 1.62 & $36.07 * *$ \\
\hline A $22 \times A 7.7$ & $10.55^{*}$ & $-19.21 * *$ & $11.10^{*}$ & A $22 \times A 7.7$ & $7.70^{*}$ & $-9.18 * *$ & $-3.19 * *$ & A $22 \times A 7.7$ & $14.88 * *$ & $7.26^{*}$ & $26.85 * *$ \\
\hline $\mathrm{A} 22 \times \mathrm{A} 13.1$ & $13.47 * *$ & $-8.63^{*}$ & 4.03 & $\mathrm{~A} 22 \times \mathrm{A} 13.1$ & $8.81^{* *}$ & -5.20 & 0.57 & $\mathrm{~A} 22 \times \mathrm{A} 13.1$ & $16.48 * *$ & 5.59 & $31.45 * *$ \\
\hline
\end{tabular}

Mid-parent heterosis $=\mathrm{H}_{t}$, Better-parent heterosis $=\mathrm{H}_{\mathrm{b}}$ 
Positive and significant heterosis over mid, better and commercial was observed for crosses $\mathrm{A} 8 \times \mathrm{C} 2.19, \mathrm{~A} 8 \times \mathrm{A} 22$, $\mathrm{A} 18.6 \times \mathrm{A} 14.1, \mathrm{~A} 22 \times \mathrm{A} 7.7, \mathrm{~A} 18.6 \times \mathrm{A} 7.7, \mathrm{~A} 14.1 \times \mathrm{A} 7.7$ and A18.6 $\times$ A22. These hybrids might act as potential source for the improvement of sunflower hybrids for yield related traits. The information about genetics of heterosis is helpful in designing the breeding strategies to meet the requirements of ever expanding population. The implication of the proposed over dominant model would be a substantial genetic advance in yield upon increasing the genetic diversity of the parental breeding material. In majority of the crosses, the expression of heterosis manifestation (mid parent, better parent and commercial) in present breeding material indicated over dominance type of gene action as the genetic basis of heterosis. Pyramiding entails stacking multiple genes leading to the simultaneous expression (Joshi and Sharma, 2010). Gene pyramiding has been used as an effective approach to develop a genotype/hybrid by stacking different genes controlling the economically important traits such as yield, quality and tolerance to abiotic stresses. It is gaining considerable importance as it would improve the efficiency of plant breeding leading to the development of genetic stocks and precise development of broad spectrum resistance capabilities.

Conclusion: On the basis of the study it is concluded that head diameter, 1000-achene weight and days to complete flowering can be relied upon for selection in a breeding program for yield improvement. The lines A2.5, A8, A14.1, crosses (A8 $\times$ A14.1), (A8 $\times$ A13.1) and double crosses $(\mathrm{A} 8 \times \mathrm{C} 2.19) \times(\mathrm{A} 8 \times \mathrm{A} 7.7),(\mathrm{A} 18.6 \times \mathrm{A} 14.1) \times(\mathrm{A} 22 \times \mathrm{A} 2.5)$, showed highest mean values for most of the traits. Accessions A8, A14.1 and line A22 are found to be good general combiners and may be used for hybrid development, while good SCA was shown by crosses A $8 \times$ A14.1, A $8 \times$ A7.7 A14.1 $\times$ A7.7, A8 $\times$ A7.7 and A22 $\times$ A2.5. Crosses $\mathrm{A} 8 \times \mathrm{A} 7.7 \mathrm{~A} 8 \times \mathrm{A} 14.1, \mathrm{~A} 22 \times \mathrm{A} 2.5,(\mathrm{~A} 8 \times \mathrm{C} 2.19) \times(\mathrm{A} 8 \times \mathrm{A} 7.7)$, $(\mathrm{A} 18.6 \times \mathrm{A} 14.1) \times(\mathrm{A} 22 \times \mathrm{A} 2.5)$ were found high yielder having high values of 1000 achene weight and oil contents showing a pyramiding of the genes/QTLs governing the expression of desired traits such as achene yield per, 1000 achene weight and oil contents.

Acknowledgment: Authors acknowledge Higher Education Commission (HEC) of Pakistan for provision of funds to carry out this project.

\section{REFERENCES}

Adefris, T., H. Jayaramaiah, B.N. Jagadeesh and A. Teklewold. 2001. Correlation and causations in sunflower (Helianthus annuus L.). Mysore J. Agri. Sci. $35: 216-225$.
Burton, G.W. and E.H. Devane. 1953. Estimating heritability in tall fescue (Fescuea arundienancea) from replicated clonal material. Agron. J. 45:478-481.

Cosge, B. and N. Bayraktar. 2004. Correlation between some yield and yield components in sunflower (Helianthus annuus L.) hybrid, line and varieties. Tarim Bilimeri Dergisi 10:111-115.

Darvishzadeh, R., H.H. Maleki and A. Sarrafi. 2011. Path analysis of the relationship between yield and some related traits in diallel population of sunflower (Helianthus annuus L.) under well watered and waterstressed conditions. Aust. J. Biol. Sci. 5:674-680.

Deengra, S.K., N. Kumar, V. Kumar and R.P.S. Dhaka. 2010. Correlation and path coefficient analysis in sunflower (Helianthus annuus L.). Prog. Agric. 10:318322.

Dewey, D.R. and K.H.A. Lu. 1959. Correlations and path co-efficient analysis of component of crested wheat grass seed production. Agron. J. 51:515-518.

Folconer, D.S. and T.F.C. Mackey. 1996. Introduction to quantitative genetics, $4^{\text {th }}$ Ed. Pearson Edu. Ltd. England. pp.253-260.

Gangappa, E., K.M. Channakrishnaiah, S. Ramesh and M.S. Harini. 1997. Exploitation of heterosis in sunflower (Helianthus annuus L.). Crop Res. Hisar. 13:339-348.

Ghaffari, M., I. Farrokhi and M. Mirzapour. 2011. Combining ability and gene action for agronomic traits and oil content in sunflower (Helianthus annuus L.) using $F_{1}$ hybrids. Crop Breed. J. 1:73-84.

Hladni, N., D. Skoric, M.K. Balalic, Z. Sakac and V. Miklic. 2007. Heterosis for agronomic important traits in sunflower (Helianthus annuus L.) Helia 30:191-198.

Jockovic, M., R. Marinkovic, A.M. Jeromela, V. Radic, P. Canak and N. Hladni. 2012. Association between seed yield and some morphological characteristics in sunflower. Ratar. Povrt. 49:53-57.

Joshi, R.K. and S. Nayak. 2010. Gene pyramiding-A broad spectrum technique for developing durable stress resistance in crops. Biotechnology and Molecular Biology Review 5(3): 51-60.

Kaya, Y., G. Evci, V. Pekcan and T. Gucer. 2006. Determining the relationship between yield and yield attributes in sunflower. Turk. J. Agric. For. 31:237-244.

Kaya, Y., G. Evci, V. Pekcan, T. Gucer, S. Durak and M.I. Yilmaz. 2008. The path analysis of yield traits in sunflower. Latvian J. Agron. 11:72-76.

Kempthorne, O. 1957. An introduction to genetic statistics. John Wiley and Sons Inc, New York. Chapman and Hall Ltd., London.

Kholghi, M., I. Bernousi, R. Darvishzadeh and A. Pirzad. 2011. Correlation and path coefficient analysis of seed yield and yield related trait in Iranian confectionery sunflower populations. Afr. J. Biotech. 10:1305813063. 
Kwon, S.H. and J.H. Torrie. 1964. Heritability and interrelationship of traits of two soybean populations. Crop Sci. 4:196-198.

Machikowa, T. and O. Saetang. 2008. Correlation and path coefficient analysis on seed yield in sunflower. Suranaree J. Sci. Technol. 15:243-248.

Mijic, A., M. Krizmani, V. Guberac and S. Maric. 2006. Path coefficient analysis of several components oil yield in sunflower (Helianthus annuus L.). Agriculture 12:1115.

Skoric, D., S. Jocic and I. Molnar. 2000. General (GCA) and specific (SCA) combining abilities in sunflower. Zbornik naucnih radova Novi Sad. 6:97-105.
Steel, R.G.D., J.H. Torrie and D.A. Dicky. 1997. Principles and Procedures of Statistics: A Biometrical Approach, $3^{\text {rd }}$ Ed. McGraw Hill Book Co. Inc. New York; pp.400428.

Syed, W.H., S.M. Syed and S. Hasnain. 2004. Variability for agronomic traits in sunflower random mating populations, correlations, estimated gain from selection and correlated responses to selection. Helia 27:85-98.

Vidhyavathi, R., P. Mahalakshmi, N. Manivannan and V. Murulidharan. 2005. Correlation and path analysis in sunflower (Helianthus annuus L.). Agric. Sci. Digest 25:6-10. 